\title{
Emission Spectra of Fallback Disks around Young Neutron Stars
}

\section{Citation}

Perna, Rosalba, Lars Hernquist, and Ramesh Narayan. 2000. "Emission Spectra of Fallback Disks around Young Neutron Stars." The Astrophysical Journal 541 (1): 344-50. https:// doi.org/10.1086/309404.

\section{Permanent link}

http://nrs.harvard.edu/urn-3:HUL.InstRepos:41381699

\section{Terms of Use}

This article was downloaded from Harvard University's DASH repository, and is made available under the terms and conditions applicable to Other Posted Material, as set forth at http:// nrs.harvard.edu/urn-3:HUL.InstRepos:dash.current.terms-of-use\#LAA

\section{Share Your Story}

The Harvard community has made this article openly available.

Please share how this access benefits you. Submit a story.

Accessibility 


\title{
Emission Spectra of Fallback Disks Around Young Neutron Stars
}

\author{
Rosalba Perna ${ }^{1}$, Lars Hernquist and Ramesh Narayan \\ Harvard-Smithsonian Center for Astrophysics, 60 Garden Street, Cambridge, MA 02138
}

\begin{abstract}
The nature of the energy source powering anomalous $X$-ray pulsars is uncertain. Proposed scenarios involve either an ultramagnetized neutron star, or accretion onto a neutron star. We consider the accretion model proposed recently by Chatterjee, Hernquist \& Narayan, in which a disk is fed by fallback material following a supernova. We compute the optical, infrared, and submillimeter emission expected from such a disk, including both viscous dissipation and reradiation of $X$-ray flux impinging on the disk from the pulsar. We find that it is possible with current instruments to put serious constraints on this and on other accretion models of AXPs. Fallback disks could also be found around isolated radio pulsars and we compute the corresponding spectra. We show that the excess emission in the $\mathrm{R}$ and I bands observed for the pulsar PSR $0656+14$ is broadly consistent with emission from a disk.
\end{abstract}

Subject headings: stars: neutron - accretion, accretion disks $-X$-rays: stars

\section{Introduction}

In the past few years, the realm of astronomical objects has been enriched with the discovery of about half a dozen of the so-called anomalous X-ray pulsars (AXPs) (Mereghetti \& Stella 1995; van Paradijs, Taam \& van den Heuvel 1995). AXPs are sources of pulsed $X$-ray emission, with persistent luminosities $L_{x} \sim 10^{35}-10^{36} \mathrm{erg} \mathrm{s}^{-1}$ and soft spectra. Their periods lie in a very narrow range, between 6 and 12 seconds and their characteristic ages are of order $10^{3}-10^{5}$ years. To date, about half the known AXPs have been associated with Galactic supernova remnants. (For a recent review of the properties of AXPs, see Mereghetti 1999.)

While AXPs are almost certainly neutron stars, their properties establish them as a distinct class from binary $X$-ray pulsars and radio pulsars. Compared to binary $X$-ray pulsars, AXPs have lower luminosities and exhibit a narrow distribution of periods. Unlike young radio pulsars, AXPs have rather longer periods and appear to be radio quiet. One key to understanding these differences is to try and identify the energy source which powers the $X$-ray emission. It is quite

\footnotetext{
${ }^{1}$ Harvard Society of Fellows
} 
clear that this energy cannot be provided by rotation (as it is in radio pulsars). For values of $P$ and $\dot{P}$ that are characteristic of AXPs, the rate of loss of rotational energy is $|\dot{E}| \equiv 4 \pi^{2} I \dot{P} / P^{3} \approx 10^{32.5}$

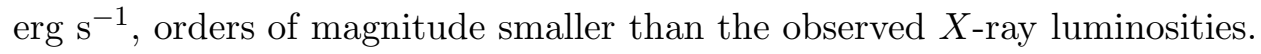

Two broad classes of models have emerged to account for the $X$-ray emission from AXPs. In one, AXPs are hypothesized to be isolated, ultramagnetized neutron stars, with field strengths in the range $10^{14}-10^{15} \mathrm{G}$, a.k.a. "magnetars" (Duncan \& Thompson 1992). Field strengths of this order are consistent with those inferred for soft gamma repeaters, on the basis of timing measurements, assuming that these objects lose rotational energy in a manner similar to radio pulsars (e.g. Kouveliotou et al. 1998, 1999). If the magnetar interpretation of AXPs is correct, the $X$-ray luminosities could be powered by either magnetic field decay (Thompson \& Duncan 1996) or by residual thermal energy (Heyl \& Hernquist 1997a,b). The observed $X$-ray luminosities place constraints on both mechanisms. If the emission is powered by residual thermal energy, then the envelope of the star must consist primarily of light elements such as hydrogen and helium (Heyl \& Hernquist 1997b). On the other hand, if the emission is powered by magnetic field decay, then a value of $B \gtrsim 10^{16} \mathrm{G}$ is required, unless non standard decay processes are invoked (Heyl \& Kulkarni 1998).

The second class of models proposes that the $X$-ray emission from AXPs is powered by accretion, and unusually high values of the magnetic field are not required. Accretion can occur from binary companions of very low mass (Mereghetti \& Stella 1995), from the debris of a disrupted high mass companion (van Paradijs, Taam \& van den Heuvel 1995; Gosh, Angelini \& White 1997) or from the interstellar medium (Wang 1997). Each of these three possibilities has been challenged by observational and theoretical considerations. Searches to detect binary companions of AXPs have failed thus far, placing severe constraints on companion masses (e.g. Wilson et al. 1998; Mereghetti, Israel \& Stella 1998). If the emission is powered by accretion from a disrupted binary companion, it is not clear why AXPs should be associated with young supernova remnants. Finally, for the specific object (RXJ 0720.4-3125) considered by Wang (1997), Heyl \& Hernquist (1998) have demonstrated that the $X$-ray luminosity can be explained by cooling, without requiring that this neutron star be old and have experienced magnetic field decay.

Recently, Chatterjee, Hernquist \& Narayan (1999; hereafter, CHN) have studied another accretion model, in which neutron stars accrete from disks which formed from fallback material after a supernova explosion. (Similar proposals have been studied recently by Alpar [1999] and Marsden et al. [1999].) The possibility that material might fall back onto a neutron star following a supernova explosion and settle in a disk is not new (e.g. Woosley 1988, Chevalier 1989). In particular, Lin, Woosley \& Bodenheimer (1991) suggested that this process might account for the presence of planets around some radio pulsars. Later, in their discussion of accretion from the debris of a disrupted companion, van Paradijs et al. (1995) noted that fallback could provide material to form disks around young AXPs, but they did not analyze this possibility in detail. 
In principle, all neutron stars could acquire debris through fallback, but subsequent accretion can occur only under specific circumstances, depending on the relative locations of the magnetospheric radius, the light cylinder radius, and the corotation radius. CHN related these conditions to physical parameters such as the initial mass of the disk, $M_{\mathrm{d}}$, the initial period of the neutron star, $P_{0}$, and the strength of the magnetic field, $B$. CHN found that neutron stars would evolve into accreting sources only for sufficiently strong magnetic fields, while objects with weaker fields would be unaffected and would, presumably, become ordinary radio pulsars. For the particular values $P_{0}=0.015$ seconds and $M_{d}=0.006 M_{\odot}$, for example, CHN found that the outcome depended on whether or not the field is greater or smaller than $B=4 \times 10^{12} \mathrm{G}$. Namely, neutron stars with $B>4 \times 10^{12} \mathrm{G}$ became AXPs, while those with $B<4 \times 10^{12} \mathrm{G}$ became radio pulsars. Other choices for $P_{0}$ and $M_{\mathrm{d}}$ yield similar behavior, and lead to different values for the critical magnetic field separating AXPs and radio pulsars.

In their analysis, CHN demonstrated that the time varying nature of accretion from a fallback disk provides a natural explanation for the fact that AXP periods increase with time. Indeed, if the accretion rate declines as $\dot{M} \propto t^{-7 / 6}$, close to the similarity solution derived by Cannizzo, Lee \& Goodman (1990), then the rate of increase of the period will be identical to spindown by magnetic dipole radiation. Thus, timing analyses of steady spindown alone cannot distinguish between these two interpretations. Moreover, in contrast with the magnetar model, the time varying accretion studied by CHN relies on a standard population of isolated neutron stars having magnetic fields similar to those inferred for radio pulsars, and therefore it does not require the existence of a separate class of neutron stars.

How can we distinguish between the time-dependent accretion interpretation and the magnetar model, given that steady spin-down is accounted for by both? One possibility may be by searching directly for disk emission, at wavelengths much longer than $X$-rays. Accretion disks dissipate energy by viscous processes at all radii, and some of this energy will be radiated at long wavelengths, especially from large radii. Moreover, the central $X$-ray emitting source irradiates the outer disk, and most of this energy is reradiated at longer wavelengths. In this paper, we compute the emission spectrum expected for accretion disks like those required to explain AXPs, and we apply the model of CHN to make predictions for the flux in various bands. The predicted flux is within the range of sensitivity of current instruments, and can be used, therefore, to directly test the accretion model. We note that while our specific estimates are made using the disk models of CHN, our arguments should apply in general to all accretion models for AXPs regardless of the source of the disk mass. Thus, sensitive observations at long wavelengths may provide powerful tests of all variants of the accretion hypothesis for AXPs.

A prediction of the CHN analysis is that fallback disks could also be found around radio pulsars, although the properties of these disks are not well-constrained. Consequently, we apply our methods to calculate long wavelength emission from disks around radio pulsars. In particular, we consider the middle-aged radio pulsar PSR0656+14, which has emission in the $\mathrm{R}$ and I bands in excess of that expected by extrapolating the thermal blackbody spectrum observed at $X$-ray 
wavelengths. We show that the excess emission is broadly consistent with that produced by an accretion disk.

\section{Model}

The evolution of the fallback material settling into a disk can be treated in a similar manner to the evolution of the accretion disk formed by material captured by a black hole after the tidal disruption of a star. Numerical simulations by Cannizzo, Lee \& Goodman (1990; hereafter CLG) show that after an initial transient phase, the evolution of the disk becomes self-similar, with the accretion rate $\dot{M}$ decreasing as a power law. Exact self-similar solutions for the evolution of the disk were derived by Pringle (1974) assuming that $\nu \propto r^{p} \sum^{q}$, where $\nu$ is the viscosity and $\Sigma$ the surface density. If the so-called $\alpha$ disk model is adopted (see CLG for details), then a solution is obtained when $p=1$ and $q=2 / 3$, yielding

$$
\nu=\operatorname{Cr} \Sigma^{2 / 3}
$$

with

$$
C \equiv \alpha^{4 / 3}\left(\frac{k_{B}}{\mu m_{p}}\right)^{4 / 3}\left(\frac{\kappa_{\mathrm{es}}}{12 a c G M}\right)^{1 / 3} .
$$

Here $\kappa_{\mathrm{es}}$ is the electron scattering opacity $2, M$ the mass of the neutron star, and $\alpha$ is the standard dimensionless viscosity constant that is expected to be $\lesssim 1$ on physical grounds (Shakura \& Sunyaev 1973). We adopt the value $\alpha=0.1$ in our numerical calculations. The corresponding surface density evolves according to

$$
\Sigma(r, t)=\Sigma_{0}\left(\frac{t}{t_{0}}\right)^{-15 / 26} f\left[\left(\frac{r}{r_{0}}\right)\left(\frac{t}{t_{0}}\right)^{-3 / 8}\right],
$$

where

$$
f(u) \equiv(28)^{-3 / 2} u^{-3 / 5}\left(1-u^{7 / 5}\right)^{3 / 2} .
$$

The constants $\Sigma_{0}, r_{0}$ and $t_{0}$ are constrained by the condition

$$
t_{0}^{-1}=\frac{C \Sigma_{0}^{2 / 3}}{r_{0}}
$$

The total mass of the disk decreases with time as

$$
M_{\mathrm{d}}(t)=(28)^{-3 / 2} \frac{4 \pi}{7} r_{0}^{2} \Sigma_{0}\left(\frac{t}{t_{0}}\right)^{-3 / 16} .
$$

\footnotetext{
${ }^{2}$ CLG considered more general opacity laws, such as the Kramers opacity, with $\kappa(\rho, T)=\kappa_{0} \rho T^{-7 / 2}$, but they found their results to be relatively insensitive to the particular expression they chose. Therefore, for simplicity we adopt a constant opacity, $\kappa_{\text {es }}$.
} 
The accretion rate is then given by $\dot{M}_{\mathrm{d}}(t)=(-3 / 16) M_{\mathrm{d}}(t) / t$. We define the initial mass of the disk to be $M_{\mathrm{d}}^{0} \equiv M_{\mathrm{d}}\left(T_{d}\right)$, where $T_{d}$ is of order the dynamical time of the disk early on. Following CHN, we select $T_{d}=1$ msec. Having chosen a value for $r_{0}$, the constant $\Sigma_{0}$ is then derived from equation (6) at $t=T_{d}$. Using the relation (5), we then find

$$
\Sigma_{0} \approx 155\left(M_{\mathrm{d}}^{0}\right)^{8 / 7}\left(T_{d} C\right)^{3 / 14} r_{0}^{-5 / 2} .
$$

Notice that, with the choice (7), (i.e. $\Sigma_{0} \propto r_{0}^{-5 / 2}$ ), the self-similar solution for $\Sigma$, as given by Eq. (3), becomes independent of the numerical value of the parameter $r_{0}$, and therefore is solely determined by $M_{\mathrm{d}}^{0}$ and $T_{d}$. However, as Equation (đ) shows, the dependence on $T_{d}$ is much weaker than the dependence on $M_{\mathrm{d}}^{0}$. Following CHN, we keep the value of $T_{d}$ fixed and use only $M_{\mathrm{d}}^{0}$ as a parameter.

The solution (3) extends from $u=0$ to $u=1$. However, an accretion disk can exist only for radii beyond the magnetospheric radius $R_{m} \approx 6.6 \times 10^{7} B_{12}^{4 / 7} \dot{m}^{-2 / 7}$ (Frank, King \& Raine 1992), where $B_{12} \equiv B /\left(10^{12} G\right)$ and $\dot{m} \equiv \dot{M} / \dot{M}_{E}$ is the mass accretion rate in Eddington units. We therefore take the minimum radius of the disk to be $r_{\min }=R_{m}$. Similarly, whereas the mathematical solution sets the disk outer boundary at $u=1$ (corresponding to $\Sigma=0$ ), in practice a disk can extend only up to a radius $r_{\max }\left(\Sigma_{\min }\right)$, where densities $\Sigma \lesssim \Sigma_{\min }$ will be destroyed by the ram pressure from the interstellar medium. We assume that a disk can exist as long as the incident flux is not sufficient to significantly perturb the angular momentum of the matter orbiting in it. We find that this condition is satisfied for densities $\Sigma \gtrsim \Sigma_{\min } \approx 5 \times 10^{-7} r_{14}^{2} M_{1.4}^{-1} n_{0} v_{300}^{2} \mathrm{~g} \mathrm{~cm}^{-2}$ where $r_{14} \equiv r /\left(10^{14} \mathrm{~cm}\right), M_{1.4} \equiv M / 1.4 M_{\odot}, n_{0} \equiv n /\left(1 \mathrm{gm} / \mathrm{cm}^{3}\right)$ is the particle density in the interstellar medium, and $v_{300} \equiv v / 300 \mathrm{~km} / \mathrm{sec}$. Therefore interstellar ram pressure affects the outer boundary of the disk only very marginally.

At any position and time, the effective temperature of the disk due to viscous dissipation is given by (CLG)

$$
T_{\mathrm{eff}}(r, t)=\left(\frac{9 \nu(r, t) \Sigma(r, t) G M}{8 \sigma r^{3}}\right)^{1 / 4} \approx\left(-\frac{3 \dot{M}_{\mathrm{d}}(t) G M}{8 \pi \sigma r^{3}}\right)^{1 / 4} .
$$

Besides emission due to internal energy dissipation, a considerable amount of flux from the disk can arise as a result of reradiation of $X$-rays impinging from the central object. Vrtilek et al. (1990) derive an analytical expression for the effective temperature of an irradiated disk, under the assumption that the disk height $h \propto r^{n}$ and irradiation is the dominant form of heating. If $L_{X}$ is the $X$-ray luminosity of the central source, and $\omega \equiv \sqrt{G M / r^{3}}$ is the Keplerian rotation frequency of the disk at position $r$, then $n=9 / 7$, and the temperature profile is given by

$$
T_{X}(r)=\left[f \frac{\sqrt{k_{B} / \mu m_{\mathrm{H}}} L_{X} \omega}{14 \pi \sigma G M}\right]^{2 / 7} \simeq 23200\left(\frac{f}{0.5}\right)^{2 / 7}\left(\frac{L_{X}}{L_{E}}\right)^{2 / 7}\left(\frac{R_{\odot}}{r}\right)^{3 / 7}
$$

\footnotetext{
${ }^{3}$ Large variations in the choice of $T_{d}$ would lead to the same numerical results with small adjustments in $M_{\mathrm{d}}^{0}$.
} 
The last equation is for $M=1.4 M_{\odot}$. Here, $f$ is a factor which contains the uncertainty in the disk structure (including the absorbed fraction of radiation impinging on the surface). We adopt the estimate given by Vrtilek et al. (1990) of $f \sim 0.5$. In the cases that we consider, irradiation typically dominates the spectrum for $L_{X} \gtrsim 10^{34} \mathrm{erg} \mathrm{s}^{-1}$; for lower values of $L_{X}$ (but generally higher than $\sim 10^{31} \mathrm{erg} \mathrm{s}^{-1}$ ), the inner parts of the disk (where the optical emission is mostly generated) are dominated by viscous heating, while irradiation dominates in the outer regions of the disk (where most of the longer-wavelength emission comes from). Therefore it is reasonable to keep the approximation (9) also in these cases and compute the contribution to the flux of the two terms separately.

The total flux is obtained by integrating the emissivity over the entire surface of the disk:

$$
F=\frac{1}{d^{2}} \int_{r \min }^{r \max } d r r \int_{0}^{2 \pi} d \phi\left[B_{\nu}\left(T_{\mathrm{eff}}\right)+B_{\nu}\left(T_{\mathrm{X}}\right)\right] \times[\cos \phi \sin \beta(r) \sin \alpha+\cos \beta(r) \cos \alpha] .
$$

Here $\alpha$ is the angle between the line of sight and the normal to the disk at $r=0$, and $\beta(r)=d h(r) / d r$ is the tilt angle of the surface of the disk, which we determine at every position in the disk from the equation of hydrostatic equilibrium in the z-direction.

The opacity in disks made of fallback material is likely to be higher than in disks with cosmic abundance, due to the expected high metal content. Following Lin, Woosley \& Bodenheimer (1991), we adopt an opacity of $\sim 10^{3} \mathrm{~cm}^{2} \mathrm{~g}^{-1}$ in our calculations. Notice, however, that in our model the predicted spectrum is insensitive to the precise value of the opacity, as the temperature is independent of it. The size of the disk is slightly larger for high $\kappa_{\mathrm{es}}$, but this has little influence on the final result. What is important, however, is that a high opacity ensures that the disk is optically thick at all radii, and therefore the emission can be computed as blackbody. A more significant uncertainty derives from the possible existence of thermally unstable regions in the disk. For disks with cosmic abundance, the unstable temperature ranges have been studied in detail (i.e. Cannizzo 1993 and references therein). For high metallicity disks the situation is likely to be quite different, as cooling depends on the abundances.

\section{Emission from disks around AXPs and radio pulsars}

In the model that we consider $(\mathrm{CHN}), \mathrm{AXPs}$ and radio pulsars are assumed to be drawn from the same underlying population of neutron stars. In one example considered by CHN, for a fallback disk with mass $M_{\mathrm{d}}^{0}=0.006 M_{\odot}$, and an initial period $P_{0}=15 \mathrm{msec}$, the evolution of

the accreting disk will lead to an AXP phase if the magnetic field of the neutron star is $B_{12} \gtrsim 4$. Smaller values of the magnetic field would lead to the more common radio pulsar phase. In the following, we consider these two situations separately, and compute the emission spectrum from the disk at various ages. 


\subsection{AXPs}

Here we consider a disk of mass $M_{\mathrm{d}}^{0}=0.005 M_{\odot}$ at an angle of $60^{\circ}$ with respect to the line of sight, and a magnetic field $B_{12}=8$ for the neutron star. According to CHN, such objects will go through a phase of accretion-induced $X$-ray emission and will appear as AXPs. We describe the $X$-ray luminosity as

$$
L_{X}=\left\{\begin{array}{ll}
\dot{m} L_{\mathrm{E}} & \text { if } \dot{m} \geq 0.01 \\
100 \dot{m}^{2} & \text { if } \dot{m} \leq 0.01
\end{array} .\right.
$$

The rapid decline of the luminosity for $\dot{m}<0.01$ is to allow for the fact that the system is likely to switch to an ADAF phase, at which point the accretion luminosity $L_{X}$ decreases faster than $\dot{M}$ (Narayan \& Yi 1995; Esin et al 1997). Some studies have suggested, in fact, that in this phase much of the mass could be ejected prior to reaching the surface of the star (e.g. Blandford \& Begelman 1999, Menou et al. 1999, Quataert \& Narayan 1999). The transition radius between the ADAF and the disk is not well determined. Moreover, it is likely to depend on the metallicity of the gas in the disk, which is very far from the standard value. For simplicity, we keep the transition radius equal to the Alfven radius (which at later times is on the order of a few thousand Schwarzschild radii). We will discuss the consequences of this uncertainty on our results.

Figure 1 shows the unextincted flux at different ages from an AXP at a distance of $5 \mathrm{kpc}$. The peak in the submillimeter range is due to the effect of irradiation. At early times $\left(T \lesssim 10^{4}\right.$ $y r$ ), irradiation dominates over viscous dissipation in the entire disk. At later times, when the $X$-ray flux is much lower, viscous heating becomes dominant in the inner parts of the disk, and this produces an enhancement in the emission in the optical region of the spectrum. The model predicts a flux of a few mJy at frequencies around $10^{12} \mathrm{~Hz}$, which is within the range of the SCUBA instrument. The infrared emission is also generally above the sensitivity limit of NICMOS on the Hubble Space Telescope. In the $\mathrm{J}$ band $(1.1 \mu \mathrm{m})$, the limiting magnitude for a $5 \sigma$ detection in 60 minutes of exposure is mag $\sim 25$, which corresponds to a flux of $\sim 10^{-4} \mathrm{mJy}=10^{-30} \mathrm{erg}$ $\mathrm{cm}^{-2} \mathrm{~s}^{-1} \mathrm{~Hz}^{-1}$.

Presently, about half a dozen AXPs are known. In Table 1 we summarize the properties of the nearest ones, and compute the expected emission in various bands, assuming that the accretion model is correct. As an illustration, we take $M_{\mathrm{d}}^{0}=0.005 M_{\odot}, B_{12}=8$, and an inclination angle of the disk $i=60^{0}$ in all cases. $X$-ray luminosity, age and distance are taken from observations 7 , and extinction by dust has been taken into account based on the measured column density of hydrogen and an average extinction for the Galaxy following Pei (1992), we fit the extinction curve by

$$
A(\lambda)=k \xi(\lambda)\left(\frac{N_{\mathrm{H}}}{10^{21} \mathrm{~cm}^{-2}}\right)
$$

\footnotetext{
${ }^{4}$ The observed $X$-ray luminosity is consistent with the value predicted by the accretion model of CHN.

${ }^{5}$ Needless to say, given the uncertainties in both theoretical and measured parameters, the numbers in the table must be simply considered as rough estimates
} 
where

$$
\xi(\lambda)=\sum_{i=1}^{6} \frac{a_{i}}{\left(\lambda / \lambda_{i}\right)^{n_{i}}+\left(\lambda_{i} / \lambda\right)^{n_{i}}+b_{i}} .
$$

Here $a_{i}, b_{i}, n_{i}, \lambda_{i}$ are fitting parameters (that can be found in Pei 1992), and $k$ is the dust-to-gas ratio. For the Galaxy, $k=0.78$.

Observations in some bands have already been made for a couple of the objects in the table. In the case of $1 \mathrm{E} 2259$, our predicted flux is below the upper limits set by Coe \& Pighling (1998) in the $\mathrm{J}$ band $(z 19.6)$, and is somewhat higher than the limit of $\sim 18$ mag set in the $\mathrm{K}$ band. For the case of $4 \mathrm{U} 01412+615$ reported by Mereghetti (1999), we get a flux comparable to the flux limit of 25 mag in the $\mathrm{V}$ band, and somewhat higher than the limit of 17 mag in the $\mathrm{K}$ band. We need to point out, however, that in our model the flux in the higher energy part of the spectrum is rather sensitive to the choice of $r_{\text {min }}$, which we take equal to the Alfven radius. If the inner bound of the disk is at a radius larger than this, then the optical and near infrared emission will be lower. The submillimeter flux, on the other hand, is produced at large radii, and therefore is quite insensitive to the specific value of $r_{\min }$. All fluxes are sensitive to $M_{\mathrm{d}}^{0}$, for which we have assumed the value $0.005 M_{\odot}$ suggested by CHN. It is unclear how much freedom is available in this parameter, but it is probably not very large. Another uncertainty is the inclination angle of the disk, for which we have assumed the value $i=60^{\circ}$. Furthermore, the distances to most of these objects are not well constrained. Given all these uncertainties, more and deeper observations in several bands are needed before drawing definitive conclusions.

Observations in the optical ( $\mathrm{R}$ and $\mathrm{B}$ band) have been made for an object not in the table, RXJ 0720.4-3125, which shares some characteristics with the known AXPs (Kulkarni \& van Kerkwijk 1998), such as a long period $(8.39 \mathrm{sec})$, but which has a rather low luminosity $\left(4 \times 10^{32}\right.$ ergs $\mathrm{s}^{-1}$ ); there is no agreement on whether it belongs to the class of AXPs. There is a claimed detection of this source in $\mathrm{R}$ and $\mathrm{B}$, but it is unclear whether the observed emission is from RXJ 0720 or if it is instead due to a background star. If it is the latter, then, for the estimated distance of $400 \mathrm{pc}$, the accretion model of CHN requires the age of this object to be $\gtrsim 2 \times 10^{6} \mathrm{yr}$ for the emission to be below the detected values. Since no $\dot{P}$ has been measured, there is no other constraint on the age. If we assume that the optical emission does come from RXJ 0720, then the CHN disk model would require an age around $2 \times 10^{6}$ yr to produce an emission in roughly the same range. The slope of the spectrum, however, is not consistent with the disk model; the model predicts that the $\mathrm{R}$ band should have higher flux than the $\mathrm{B}$ band, while the observations show the contrary. Observations in other bands are needed to confirm the spectral slope 0 .

\footnotetext{
${ }^{6}$ Note, incidentally, that also in the case of PRS $0656+14$ (Fig. 3), the flux in the B band does not follow the trend of the other data points.
} 


\subsection{Radio pulsars}

In the accretion picture, AXPs and pulsars are drawn from the same population, and thus it is reasonable to expect that disks from fallback material should also be found around some pulsars. Figure 2 shows the emission spectrum from a disk surrounding a pulsar at various ages. The $X$-ray luminosity of the star at each age has been taken from the cooling curve for a neutron star with an iron envelope and $B=0$ computed by Heyl \& Hernquist (1998) \. In all cases, we chose $B_{12}=3$, and a disk mass equal to $M_{\mathrm{d}}=0.005 M_{\odot}$ at an inclination angle of $60^{\circ}$, as for AXPs; however, whereas there is a relatively tight constraint on the specific value of the disk mass for AXPs, the disk mass could be much lower (or indeed even zero) for radio pulsars. Thus, our predictions are less firm.

We have applied our model to PSR 0656+14. This is an isolated, middle-aged pulsar which shows an enhanced emission redward of the V band (Pavlov, Welty \& Cordova 1997). The data are shown in Figure 3. The soft X-ray part of the spectrum is well fitted by a thermal component, originating from the surface of the neutron star. This part of the spectrum looks similar to that observed in other pulsars, such as Geminga. As Pavlov et al. notice, however, the excess emission in the $\mathrm{R}$ and $\mathrm{I}$ bands is not very common, and they fitted it with a non-thermal component assumed to be produced in the magnetosphere. Here we consider the possibility that, whereas the high energy emission comes from the surface of the star (and is well fitted by a Rayleigh-Jeans spectrum), the low-energy emission could be produced by a residual disk. Following the notation of Pavlov et al. for the Rayleigh-Jeans part, we fit the spectrum as the sum of these two components:

$$
f(\nu)=\left[F_{\text {disk }}(\nu)+g_{0}\left(\frac{\nu}{\nu_{0}}\right)^{2}\right] \times 10^{-0.4 A(\nu)},
$$

where $\nu_{0}$ is an arbitrary reference frequency (which we took equal to $5.8 \times 10^{14} \mathrm{~Hz}$ ), $g_{0}=3.12 \times 10^{-31} G$ ergs cm ${ }^{-2} \mathrm{~s}^{-1} \mathrm{~Hz}^{-1}$, and $G \equiv T_{6}\left(R_{10} / d_{500}\right)^{2}$. Given the age $\tau=1.1 \times 10^{5} \mathrm{yr}$ (Pavlov, Stringfellow \& Cordova 1996), a distance of 760 pc (Taylor, Manchester \& Lyne 1993), an extinction of $A_{\mathrm{V}}=0.15$ and $E(B-V)=0.05$ (Pavlov, Stringfellow \& Cordova 1996), a magnetic field $B_{12}=4.7$, and an $X$-ray luminosity of $3.5 \times 10^{32}(D / 760 \mathrm{pc})^{2}\left(F / 5 \times 10^{-12} \mathrm{ergs}^{-1} \mathrm{~cm}^{-2} \mathrm{~s}^{-1}\right)$ $\operatorname{erg~s}^{-1}$ (Cordova et al. 1989, Finley, Ögelman \& Kiziloğlu 1992), the only free parameters of the disk model are the disk mass and the inclination angle of the disk. Figure 3 shows the resulting spectrum corresponding to three choices of these parameters. Notice, however, that the observations imply rather low values of $\dot{M}$, and the best fit is obtained for an extreme value of the inclination angle.

If the assumption of an accretion disk surrounding this pulsar is correct, then it should be possible to observe it directly. Figure 4 shows the predicted spectrum over a wide range of

\footnotetext{
${ }^{7}$ In the model made by Heyl \& Hernquist, various cooling curves, corresponding to different choices of the composition of the envelope of the neutron star and strength of its magnetic field, cross around $10^{5}$ yr. Therefore, except for either very young or very old systems, our results are not very sensitive to this particular choice.
} 
wavelength. The emission in the submillimeter is within the range of sensitivity of SCUBA.

\section{Conclusions}

We have computed the emission from time-dependent accretion disks around neutron stars, established by fallback following a supernova explosion. Such disks have been proposed as candidates for the energy source of AXPs. We applied our model to predict the emission in the optical, infrared and submillimeter bands for the known AXPs. The predicted fluxes are within the range of sensitivity of current instruments. We need to emphasize, however, that whereas our specific estimates have been made using the disk models of $\mathrm{CHN}$, the arguments should apply in general to any accretion model for AXPs regardless of the source of the disk mass. Therefore, deep observations at long wavelengths can be used to test all variants of the accretion hypothesis for AXPs.

If accretion disks are indeed responsible for powering the $X$-ray emission from AXPs, then they should also be found around typical radio pulsars. We computed the emission for parameters typical of radio pulsars and showed that emission from a disk might explain the excess flux redwards of the B band observed in the pulsar PSR 0656+014. Sensitive observations at longer wavelengths would severely test this interpretation.

In addition to perhaps exploring the energy source powering AXPs, accretion disks around young compact objects may have other observable consequences. Planets have been found around at least one pulsar, PSR B1257+12 (Wolszczane \& Frail 1992; Wolszczan 1994). Fallback debris would appear to be an ideal source of planet-forming material since it would naturally be metal-rich if it is drawn from the inner regions of the progenitor star (e.g. Lin et al. 1991). In some cases, fallback may be sufficiently intense to yield a black hole remnant rather than a neutron star. Recently, a class of radio-quiet $X$-ray point sources has been identified, consisting of approximately ten members, the majority of which are associated with young supernova remnants (e.g. Brazier \& Johnston 1999). Time variability in the emission from the source in SNR RCW 103, for example, has motivated the possibility that this source may be an accreting black hole rather than a cooling neutron star (e.g. Gotthelf, Petre \& Vasisht 1999). Qualitatively, accreting disks established by fallback around black holes should be similar to those examined here. Thus, deep observations at optical, infrared and submillimeter wavelengths may also reveal the true nature of radio quiet point sources in supernova remnants.

This work was supported in part by NSF grant AST 980686.

\section{REFERENCES}

Alpar, M. A. 1999, astro-ph/9912228 
Blandford, R. D. \& Begelman, M. C. 1999, MNRAS, 303, L1

Brazier, K. T. S. \& Johnston, S. 1999, MNRAS, 305, 671

Cannizzo, J. K. 1993, ApJ, 419, 318

Cannizzo, J. K., Lee, H. M. \& Goodman, J. 1990, ApJ, 351, 38 (CLG)

Chatterjee, P., Hernquist, L. \& Narayan, R. 1999, ApJ, submitted (CHN)

Chevalier, R. 1989, ApJ, 346, 847

Coe, M. J. \& Pighling, S. L. 1998, MNRAS, 299, 233

Corbet, R. H. D., Smale, A. P., Ozaki, M., \& Koyama, K. 1995, ApJ, 443, 786

Cordova, F. A., Kartje, J. Rodriguez-Bell, T. Mason, K. O., \& Middleditch, J. 1989, ApJ, 345, 451

Duncan, R.C. \& Thompson, C. 1992, ApJ, 392, L9

Esin, A. A., McClintock, J. E., \& Narayan, R. 1997, ApJ, 489, 865

Finley, J. P. Ögelman, H. \& Kiziloğlu, Ü. 1992, ApJ, 394, L21

Frank, J. King, A. \& Raine, D. 1992, "Accretion Power in Astrophysics" (Cambridge, Cambridge Univ. Press)

Gosh, P., Angelini, L. \& White, N. E. 1997, ApJ, 478, 713

Gotthelf, E. V. \& Vasisht, G. 1997, ApJ, 486, L123

Gotthelf, E. V., Petre, R. \& Vasisht, G. 1999, ApJ, 514, L107

Heyl, J. S. \& Hernquist, L. 1997a, ApJ, 491, L95

Heyl, J. S. \& Hernquist, L. 1997b, ApJ, 489, L67

Heyl, J. S. \& Hernquist, L. 1998, MNRAS, 297, L69

Heyl, J. S. \& Kulkarni, S. R. 1998, ApJ, 506, L61

Kouveliotou, C. et al. 1998, Nature, 393, 235.

Kouveliotou, C. et al. 1998, ApJ, 510, L115.

Kulkarni, S. R. \& van Kerkwijk, M. H. 1998, ApJ, 507, L49

Lin, D. N. C., Woosley, S. E. \& Bodenheimer, P. H. 1991, Nature, 353, 827

Marsden, D. Lingenfelter, R. E., Rothschild, R. E. \& Higgdon, J. C. 199, ApJ, submitted (astro-ph/9912207)

Mereghetti, S. 1999, astro-ph/9911252

Mereghetti, S. \& Stella, L. 1995, ApJ, 442, L17

Mereghetti, S., Israel, G. L., \& Stella, L. 1998, MNRAS, 296, 689

Narayan, R. \& Yi, I. 1995, ApJ, 452, 710 
Narayan, R., Mahadevan, R., \& Quataert, E. 1998, in Theory of Black Hole Accretion Disks, eds. M. A. Abramowicz, G. Bjornsson, \& J. E. Pringle, p148 (Cambridge: Cambridge Univ. Press)

Osterbroek, T., Parmar, A. N., Mereghetti, S., \& Israel, G. L. 1998, A\&A, 334, 925

Pavlov, G. G., Stringfellow, G. S. \& Cordova, F.A. 1996, ApJ, 467, 370

Pavlov, G. G., Welty, A. D. \& Cordova, F.A. 1997, ApJ, 489, L75

Pei, Y. 1992, ApJ, 395, 130

Pringle, J. E. 1974, Ph.D. thesis, University of Cambridge

Quataert, E. \& Narayan, R. 1999, ApJ, 520, 298

Shakura, N. I. \& Sunyaev, R. A. 1973, A\&A , 24, 337

Sugizaki, M. et al. 1997, PASJ, 49, L25

Thompson, C. \& Duncan, R. C. 1996, ApJ, 473, 322

van Paradijs, J., Taam, R. E. \& van den Heuvel, E. P. J. 1995, A\&A, 299, 41

Vrtilek, S. D., Raymond, J. C., Garcia, M. R., Verbunt, F., Hasinger, G. \& Kürster, M. 1990, A\&A, 235, 162

Taylor, J. H., Manchester, R. N. \& Lyne, A. G. 1993, ApJS, 88, 529

Wang, J. C. L. 1997, ApJ, 486, L119

Wilson, C., Dieters, S., Finger, M.H., Scott, D.M. \& van Paradijs, J. 1999, ApJ, 513, 464

Wolszczan, A. 1994, Science, 264, 538

Wolszczan, A., Frail, D. 1992, Nature, 355, 145

Woosley, S.E. 1988, ApJ, 330, 218 


\begin{tabular}{|c|c|c|c|c|c|}
\hline object & $\begin{array}{c}1 \mathrm{E} 2259+586 \\
(1)\end{array}$ & $\begin{array}{c}1 \mathrm{RXS} \mathrm{J170849-400910} \\
(2)\end{array}$ & $\begin{array}{c}4 \mathrm{U} 01412+615 \\
(3)\end{array}$ & $\begin{array}{c}1 \mathrm{E} \mathrm{1048-5937} \\
(4)\end{array}$ & $\begin{array}{c}1 \mathrm{E} \mathrm{1841-045} \\
(5)\end{array}$ \\
\hline$P / 2 \dot{P}(\mathrm{yr})$ & $1.75 \times 10^{5}$ & 8500 & $6 \times 10^{4}$ & 5000 & 4000 \\
\hline $\mathrm{D}(\mathrm{kpc})$ & 4 & 10 & 4 & 10 & 7 \\
\hline$N_{\mathrm{HI}}\left(\mathrm{cm}^{-2}\right)$ & $9 \times 10^{21}$ & $1.4 \times 10^{22}$ & $10^{22}$ & $10^{22}$ & $2 \times 10^{22}$ \\
\hline$L_{X}(\mathrm{erg} / \mathrm{sec})$ & $8 \times 10^{34}$ & $1.2 \times 10^{36}$ & $10^{36}$ & $5 \times 10^{35}$ & $3.5 \times 10^{35}$ \\
\hline \hline$F_{\nu_{1}}(\mathrm{mJy})$ & 30 & 5 & 90 & 3 & 4 \\
\hline$F_{\nu_{2}}(\mathrm{mJy})$ & 0.1 & 0.4 & 1 & 0.1 & 0.1 \\
$\mathrm{~K}(\mathrm{mag})$ & $\sim 17$ & $\sim 16$ & $\sim 15$ & $\sim 17$ & $\sim 17$ \\
\hline$F_{\nu_{3}}(\mathrm{mJy})$ & $10^{-4}$ & $6 \times 10^{-5}$ & $2 \times 10^{-3}$ & $2 \times 10^{-4}$ & $2 \times 10^{-6}$ \\
$\mathrm{~V}(\mathrm{mag})$ & $\sim 26$ & $\sim 27$ & $\sim 24$ & $\sim 26$ & $\sim 28$ \\
\hline
\end{tabular}

Table 1. - Predicted flux at frequencies $\nu_{1}=2000 \mathrm{GHz}, \nu_{2}=1.36 \times 10^{14} \mathrm{~Hz}$ (K band), $\nu_{3}=5.4 \times 10^{14} \mathrm{~Hz}$ (V band) for some of the known AXPs. The model parameters adopted here are the same as in Figure 1. References for these objects can be found in Corbet et al. (1), Sugizaki et al. (2), Wilson et al. (3), Osterbroek et al. (4), Gotthelf \& Vasisht (5). 


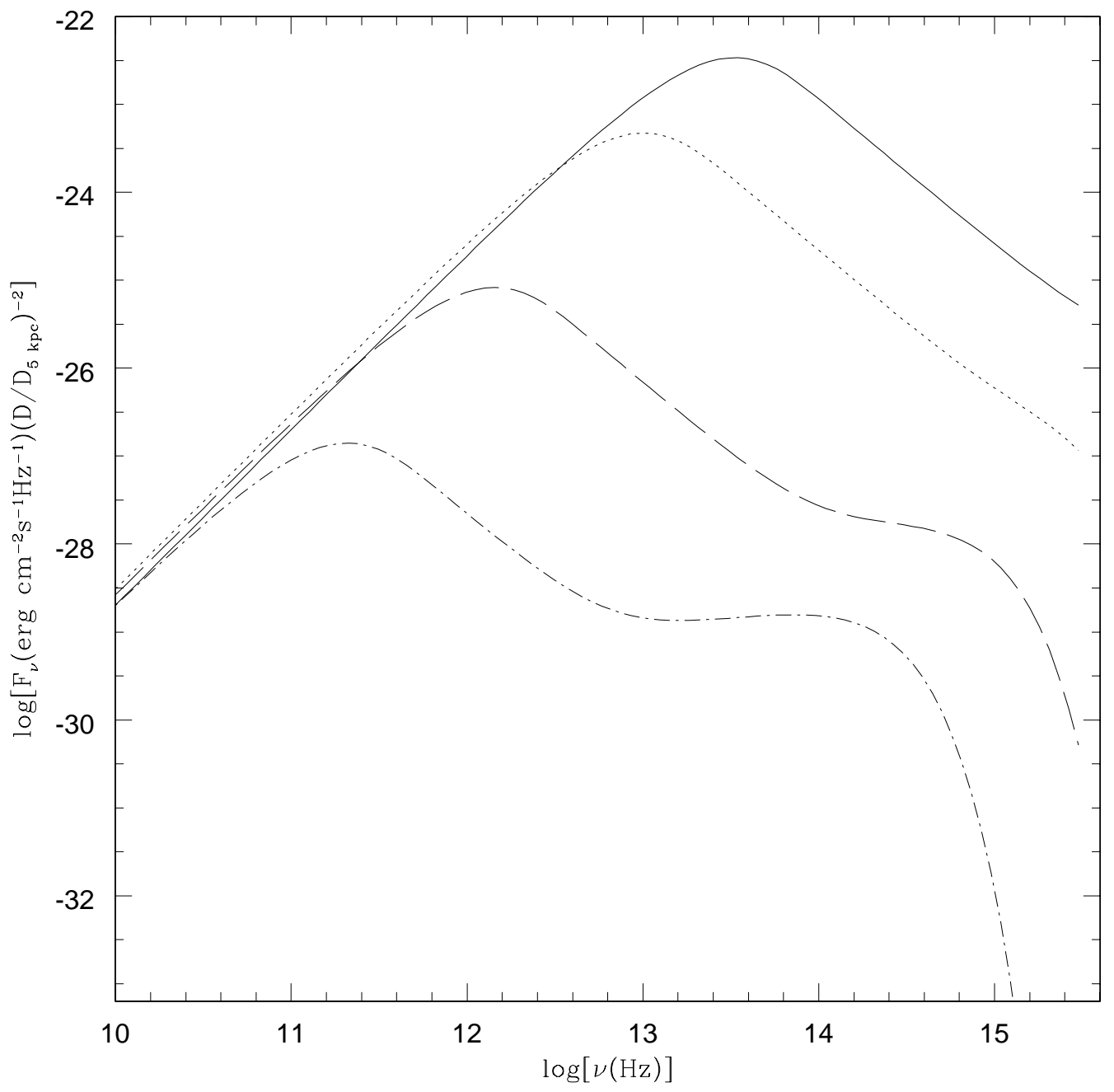

Fig. 1. - Emission spectrum from an accretion disk around an AXP at different ages. The disk mass is $M_{\mathrm{d}}^{0}=0.005 M_{\odot}$ and the magnetic field strength is $B=8 \times 10^{12} \mathrm{G}$, similar to values considered in the accretion model of CHN. The irradiating $X$-ray flux is assumed to derive from accretion onto the neutron star. The four curves correspond to ages of $\tau=10^{3} \mathrm{yr}$ (solid line), $\tau=10^{4} \mathrm{yr}$ (dotted line), $\tau=10^{5} \mathrm{yr}$ (dashed line), $\tau=10^{6} \mathrm{yr}$ (dotted-dashed line). The inclination angle of the disk is assumed to be $i=60^{0}$. 


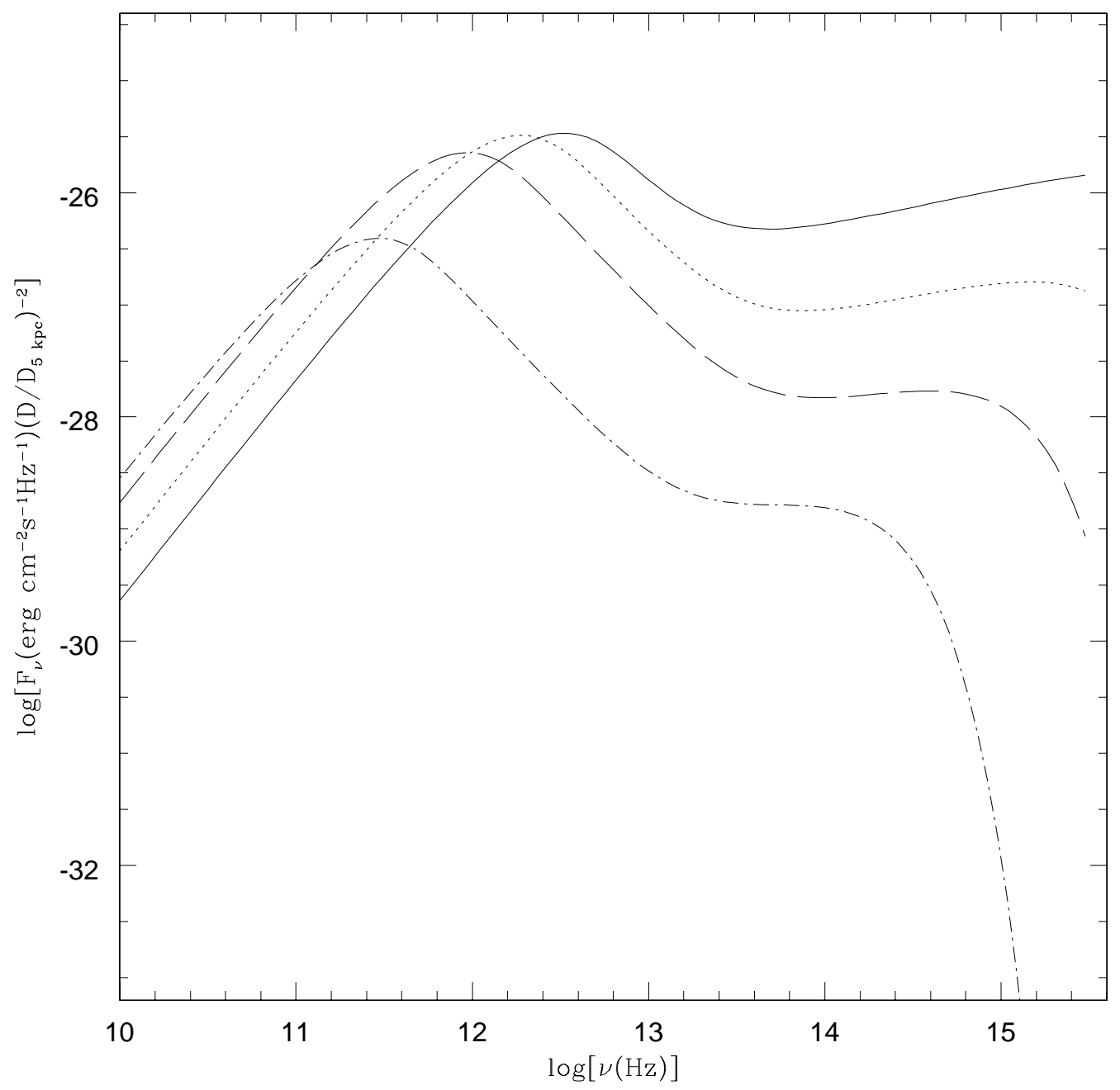

Fig. 2.- Emission spectrum from an accretion disk around a normal pulsar at different ages. Here the disk mass has the same value as in Fig. 1 for AXPs, but the magnetic field strength is smaller, $B=3 \times 10^{12} \mathrm{G}$, making accretion impossible according to the model of CHN. The irradiating $X$-ray flux is assumed to be generated by cooling of the neutron star. The four curves correspond to ages of $\tau=10^{3} \mathrm{yr}$ (solid line), $\tau=10^{4} \mathrm{yr}$ (dotted line), $\tau=10^{5} \mathrm{yr}$ (dashed line), $\tau=10^{6} \mathrm{yr}$ (dotted-dashed line). The inclination angle of the disk is assumed to be $i=60^{0}$. 


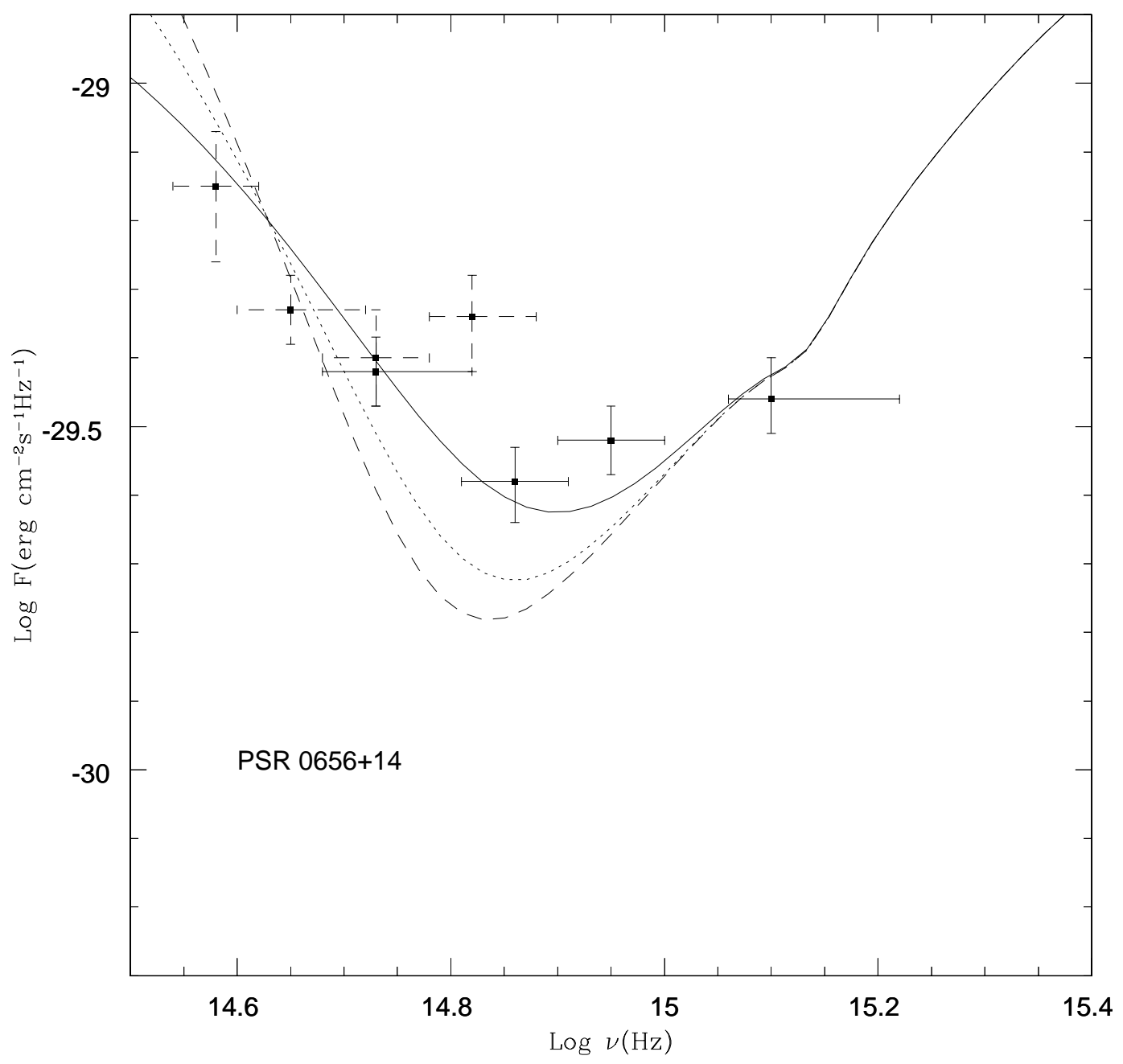

Fig. 3. - The excess flux redward of the B band observed for the pulsar PSR 0656+14 is consistent with emission from an accretion disk. Age, irradiating $X$-ray flux, dust extinction and distance are taken from observations. The various lines correspond to a model with $M_{\mathrm{d}}^{0}=0.00015 M_{\odot}, i=60^{0}$ (dashed line), $M_{\mathrm{d}}^{0}=0.00023 M_{\odot}, i=80^{0}$ (dotted line), $M_{\mathrm{d}}^{0}=0.0004 M_{\odot}, i=87^{0}$ (solid line). The emission at high energies is assumed to come from the surface of the neutron star. 


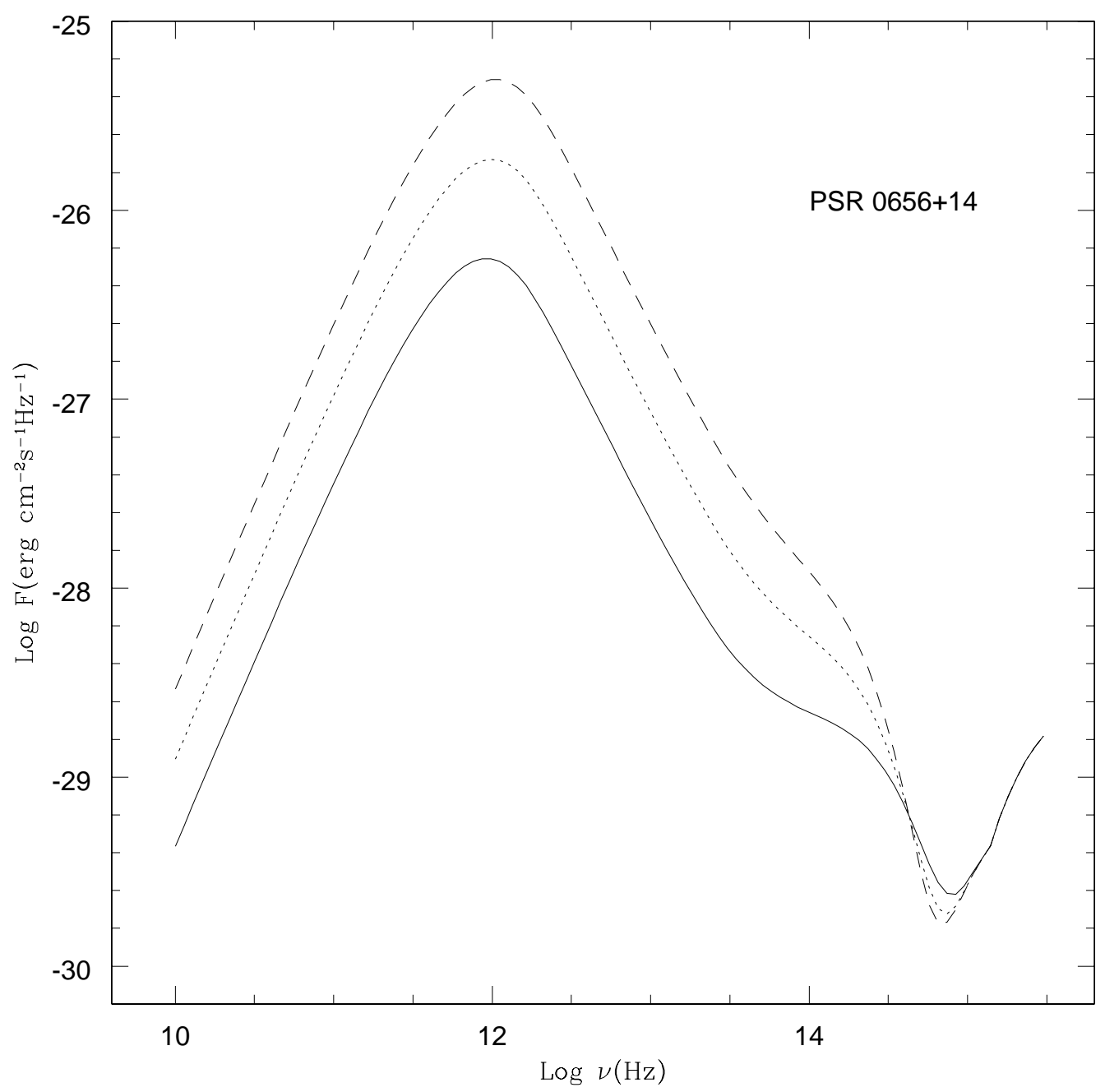

Fig. 4.- Emission spectrum from PSR 0656+14 if an accretion disk is responsible for the excess emission observed redward of the B band. The various lines correspond to the same models as in Figure 3. 